EPJ Web of Conferences 75, 02005 (2014)

DOI: 10.1051/epjconf/20147502005

(C) Owned by the authors, published by EDP Sciences, 2014

\title{
Micromagnetic simulation of a ferromagnetic particle
}

\author{
N. Ntallis and K.G. Efthimiadis \\ Department of Physics, Aristotle University, 54124 Thessaloniki, Greece
}

\begin{abstract}
In this work, the magnetic behaviour of a ferromagnetic particle has been investigated by means of micromagnetic modelling, using the Finite Element Method. The simulations were performed on an ellipsoidal particle with uniaxial magnetocrystalline anisotropy by varying the anisotropy constant, the shape and dimensions of the particle. The results indicate the critical particle size for different reversal modes. Above a critical size the formation and motion of domain walls is clearly observed. The associated nucleation and coercive fields are estimated from the demagnetization curves.
\end{abstract}

\section{Introduction}

In order to understand the magnetic behaviour of matter, the study of the response of a single magnetic element is of great importance. The shape of the element and intrinsic parameters such as the exchange length and the anisotropy constant strongly affect the magnetization loop. By varying these parameters, different reversal modes at different critical fields appear. Due to the high nonlinearity of the problem only numerical methods can show quantitative results. The Finite Element Method used in this work is a well-established technique, at least for spatial discretization, due to its capability of representing complex geometries [1].

\section{Numerical method}

The evolution of the normalized magnetization vector $\boldsymbol{m}$ is governed by the damped partial differential equation

$$
\tau \frac{\mathrm{d} \boldsymbol{m}}{\mathrm{d} t}=-\boldsymbol{m} \times\left(\boldsymbol{m} \times \boldsymbol{h}_{e f f}\right)
$$

A Rayleigh dissipation function is used to take into account the relaxation of the moment towards equilibrium [2]. $\boldsymbol{h}_{\text {eff }}$ is the normalized field intensity $\boldsymbol{H}_{\text {eff }} / M_{s} . \boldsymbol{H}_{\text {eff }}$ is the variational derivative of the free micromagnetic energy with respect to the magnetic polarization. Contributions to total energy arise from exchange, magnetocrystalline anisotropy, dipole energy and Zeeman energy [3, 4]. Simulations were performed for ellipsoidal particles with uniaxial anisotropy. $\boldsymbol{H}_{e f f}$ is described by the relation

$$
\boldsymbol{H}_{e f f}=\boldsymbol{H}_{e x t}+\boldsymbol{H}_{\text {dip }}+2 \kappa^{2}\left(\boldsymbol{m} \cdot \boldsymbol{u}_{\text {ani }}\right) \boldsymbol{u}_{\text {ani }}+\ell_{e x}^{2} \nabla^{2} \boldsymbol{m}
$$

where $\kappa=\sqrt{K_{1} / \mu_{o} M_{S}^{2}}$ is the dimensionless hardness parameter and $\ell_{e x}=\sqrt{A / \mu_{o} M_{S}^{2}}$ the exchange length. $\boldsymbol{H}_{\text {dip }}$ is the dipole field, calculated from the gradient of the magnetic scalar potential $\phi$ derived from $\nabla^{2} \phi=$ $-\nabla M$.

A thermal field $\boldsymbol{H}_{t h}$ is added, defined by a Gaussian random process in space, i.e. $\left\langle\boldsymbol{H}_{t h}^{i} \cdot \boldsymbol{H}_{t h}^{j}\right\rangle=\Gamma^{2} \delta_{i j}$. No time correlation is assumed. The amplitude $\Gamma$ is assumed to be $10^{-3} M_{S}$. The governing equation is fulfilled with the boundary condition $\partial \boldsymbol{m} / \partial \widehat{\boldsymbol{n}}=0$, where $\widehat{\boldsymbol{n}}$ denotes the outward normal of the magnetic body [3]. The equation is also normalized with respect to the time constant of the system $\tau$.

$\boldsymbol{H}_{\text {ext }}$ varies from $M_{s}$ to $-M_{s}$ in a linear manner, with a duration of $10^{6} \tau$. In this context, time integration is more an iterative procedure where the system passes from metastable states, rather than a real time simulation.

In the simulation $\boldsymbol{m}$ is described by $3^{\text {rd }}$ order Lagrange polynomials, whereas $\phi$ with $2^{\text {nd }}$ order ones. $\boldsymbol{m}$ and $\phi$ are solved simultaneously and not the one following the other.

A BDF scheme of variable step size and order is used for the time integration, and the PARDISO solver is used for the linear system of equations. As a nonlinear solver, a damped Newton's method is used. 


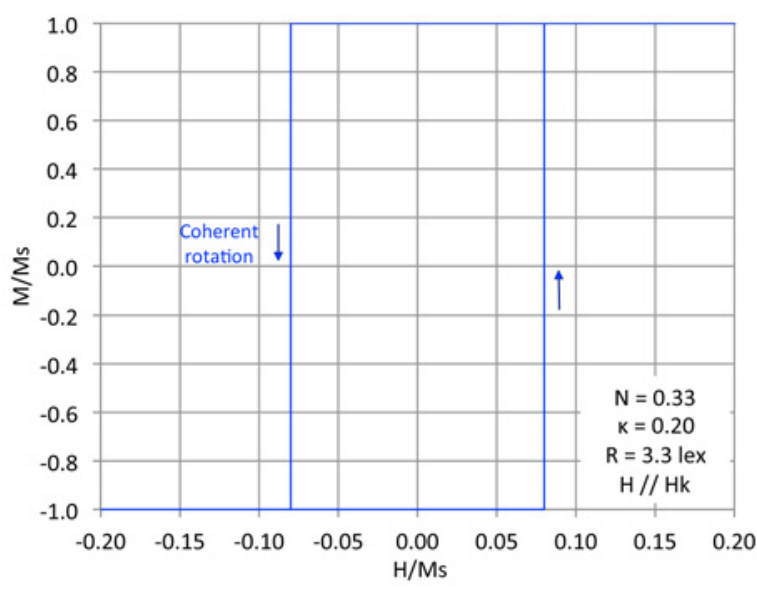

(a)

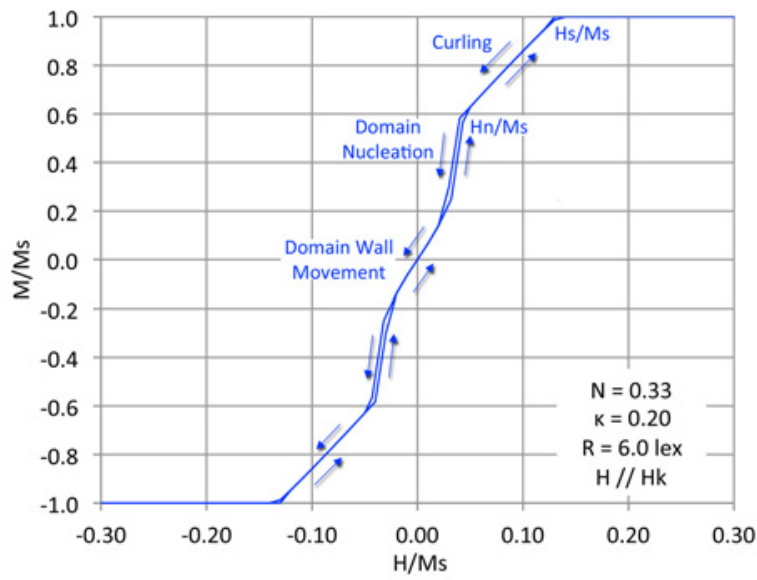

(c)

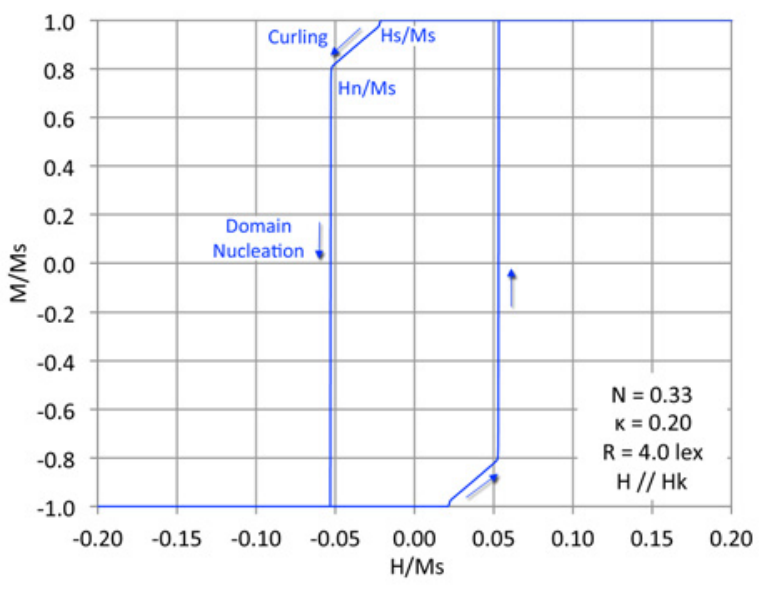

(b)

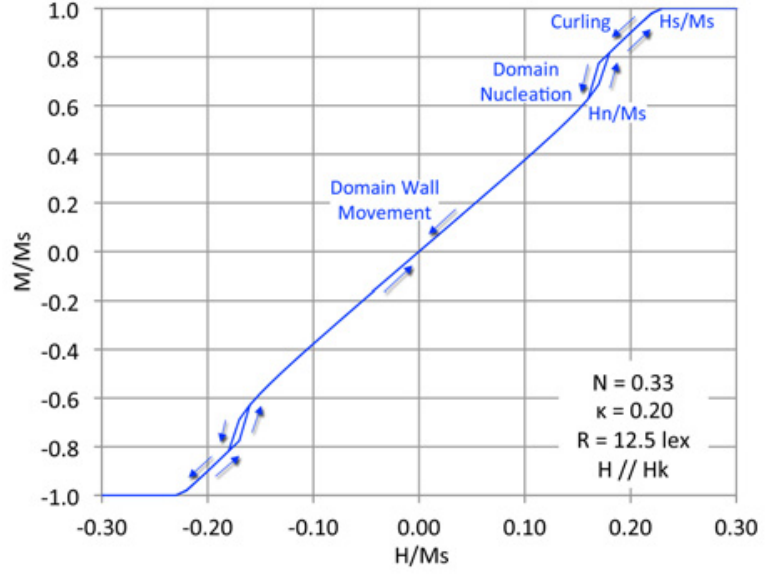

(d)

Fig. 1. Normalized hysteresis loops obtained for a spherical particle. The inlet panel shows the parameters of each loop.

The mean mesh size is kept close to the measured domain wall width, not the known Bloch wall width $\delta_{w}=\sqrt{A / K_{1}}$ [3]. Due to the BDF scheme, an error in preserving the magnetization norm occurs [5]. In this work, the error in $|\boldsymbol{m}|^{2}$ is smaller than $10^{-3}$ without any renormalization steps.

\section{Simulation results}

Figure 1 shows hysteresis loops obtained for a single spherical particle with varying radius. As the radius increases the appearance of different reversal mechanisms is observed. The results show that until a threshold radius $R_{c o h}$ is reached, the only reversal mechanism is coherent rotation.

For $R>R_{\text {coh }}$ the magnetization reversal is incoherent and always starts with a curling mode (Figure 2). At a critical field $H_{S}$ the magnetization deviates from saturation with the formation of a vortex normal to the applied field. The transverse component of $\boldsymbol{M}$ is greater at the boundaries of the particle and zero in the center of the vortex, while for the longitudinal component the opposite is observed. In most cases, the centre of the vortex coincides with the centre of the particle.
For $R>R_{\text {coh }}$ the critical field appears to obey a relation of the form

$$
\frac{H_{s}}{M_{s}}=N-2 \kappa^{2}-4.4\left(\frac{\ell_{e x}}{R}\right)^{2}
$$

while for $R \leq R_{\text {coh }}$ it is constant:

$$
\frac{H_{S}}{M_{S}}=(3 N-1) / 2-2 \kappa^{2}
$$

In the above equations $N$ is the demagnetization factor. The same relation was proposed by Kronmüller and Fänhle [6], but with different coefficients.

This relation holds for all the ellipsoids under study (Figure 3). The intersection of the two equations gives the $R_{c o h} / \ell_{e x}$ ratio, which is independent from the anisotropy constant. Note that, for a spherical particle this ratio is smaller than the one reported in international literature [3].

Decreasing the external field below $H_{s}$ the longitudinal component gets smaller enforcing the vortex state. 


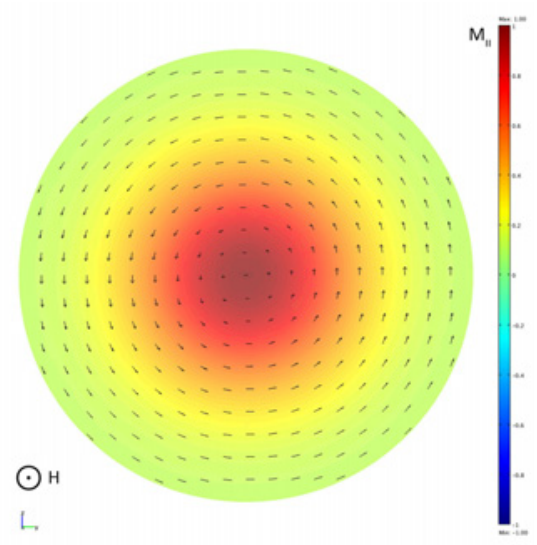

(a)

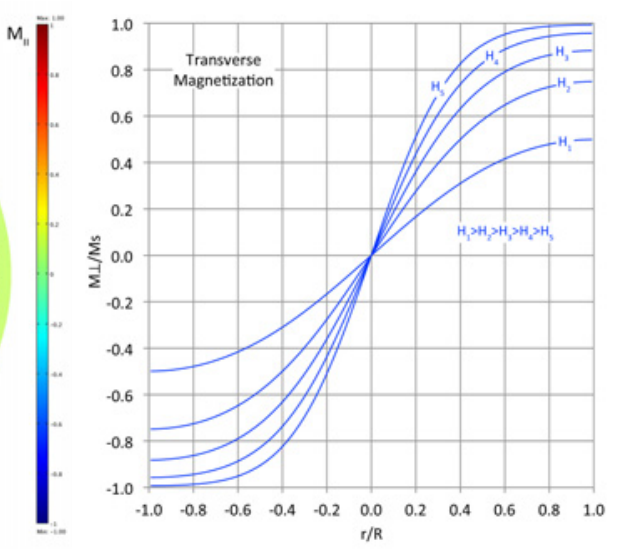

(b)

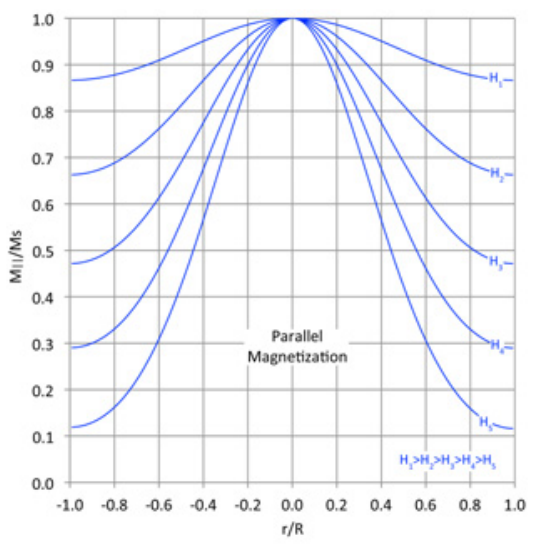

(c)

Fig. 2. The curling mode: (a) Schematic representation of the magnetization normal to the applied field (colour code is for the longitudinal component), (b) transverse and (c) longitudinal component for different applied fields.

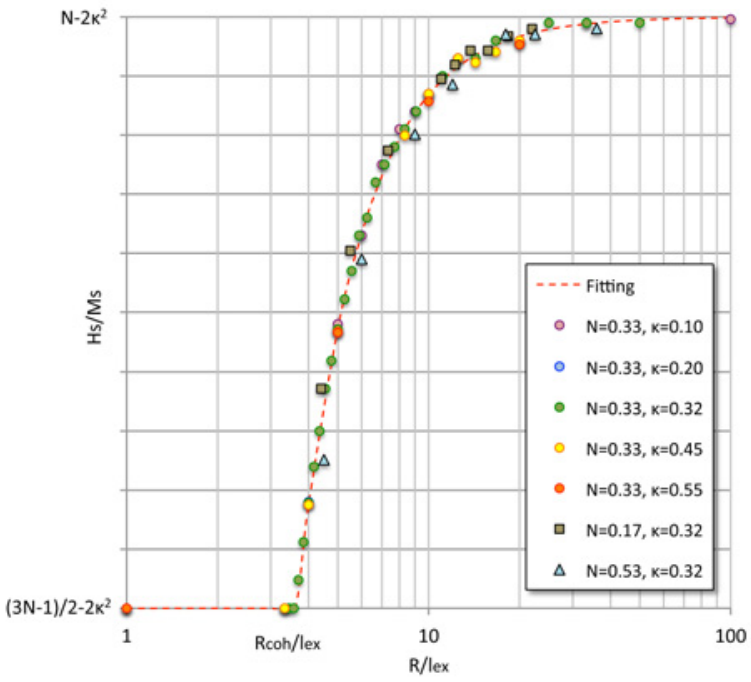

Fig. 3. $H_{s}$ vs. $R / \ell_{e x}$. The inlet panel shows the demagnetizing factor and hardness parameter.
When $H_{\text {ext }}$ reaches a critical field $H_{n}$ a second stage appears. At some point of the circumference of the particle normal to the applied field, the nucleation of a reversed domain occurs (Figure 4). The vortex state disappears and is replaced with a two domain state with a $180^{\circ}$ wall separating them. The domain wall moves until another critical field, after which the wall annihilates, ending the reversal process.

If the hardness parameter is small, the difference between $H_{s}$ and $H_{n}$ depends on $R / \ell_{e x}$ (Figure 5). For $R \approx R_{\text {coh }}$ the difference is small and the loop is almost square (Figure $1 \mathrm{~b}$ ). As $R$ increases the difference increases as well, reducing the size of the loop. For large $R$ the loop disappears, indicating a soft magnetic material and the difference tends to zero again (Figures 1c, 1d).

For a large hardness parameter, $H_{n} \approx H_{s}$ independently from the size of the particle, and nucleation begin immediately after the curling mode appears.

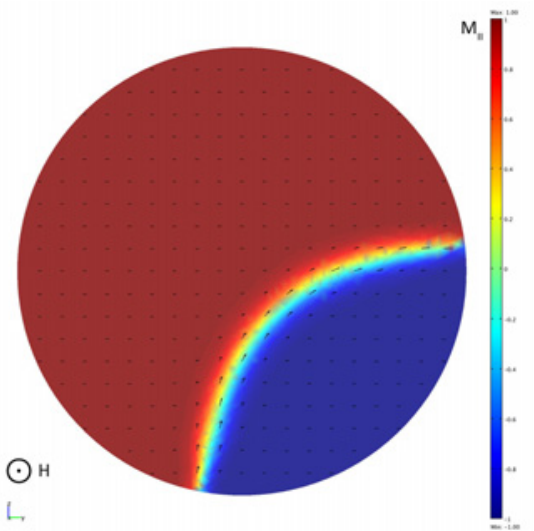

(a)

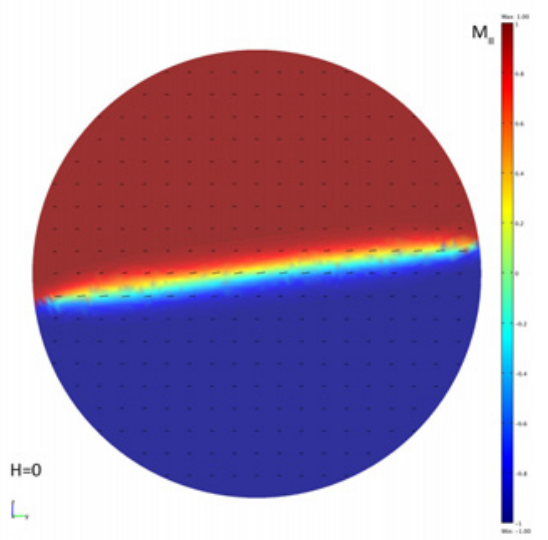

(b)

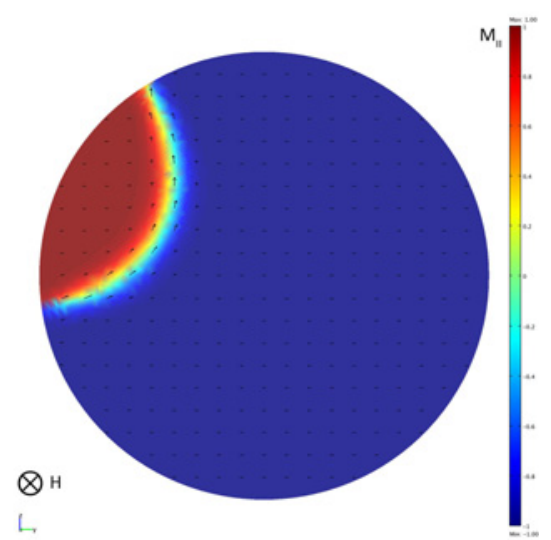

(c)

Fig. 4. Evolution of a nucleation process: (a) Nucleation of reversed domain at $H_{n}$, (b) two domain state at $H_{\text {ext }}=0$ and (c) the process just before the annihilation of the wall. The annihilation field is not equal to the nucleation field (colour code is for the longitudinal component). 


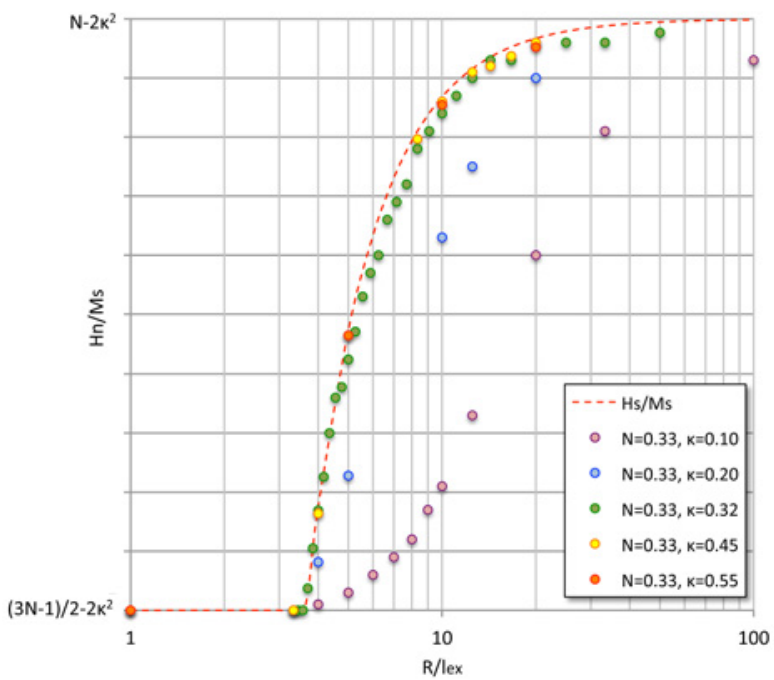

Fig. 5. $H_{n}$ vs. $R / \ell_{e x}$ for a spherical particle with different hardness parameters.

In coherent rotation, at the reversal field the magnetization difference $\Delta M=2 M_{S}$. In nucleation this difference gets smaller, as the radius of the particle increases (Figure 6). When $H_{n}$ is negative the volume of the reversed domain is almost equal to the volume of the particle. This indicates a very fast transition with a very large susceptibility. The loop is almost square, and the coercive field $H_{c}$ coincides with $H_{n}$ (Figure 7).

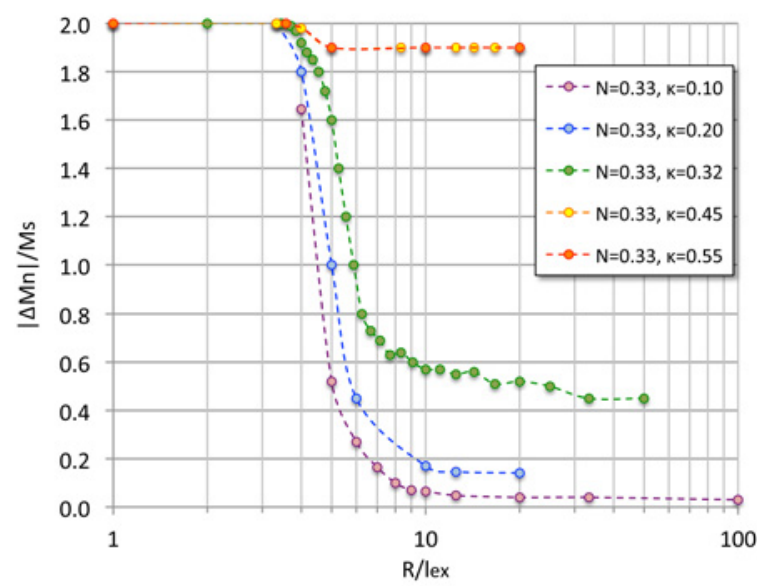

Fig. 6. Normalized magnetization difference at the nucleation field for a spherical particle.

If $H_{n} \geq 0$ the volume of the reversed domain is only a fraction of the particle's volume. A gradient of the external field is needed to move the domain wall and expand the reversed domain (Figures 1c, 1d, 4b, 4c). In this case $H_{c}$ tends to zero.

The calculated domain wall width does not obey the relation for a Bloch wall [3]. This is explained by the fact that the classical relation $\delta_{w}=\sqrt{A / K}$ does not take into account dipole interactions. The domain wall width depends also from the size of the particle (Figure 8).

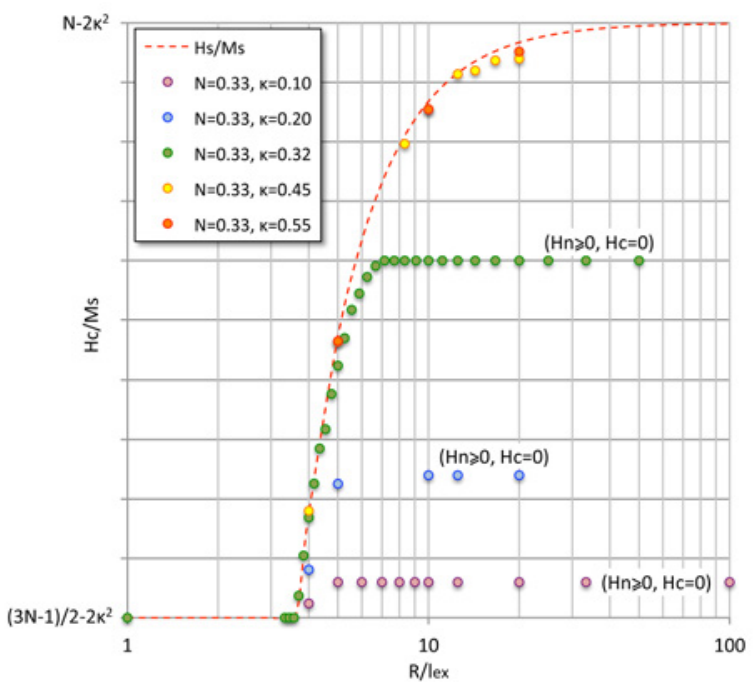

Fig. 7. $H_{c}$ vs. $R / \ell_{e x}$ for a spherical particle with different hardness parameters.

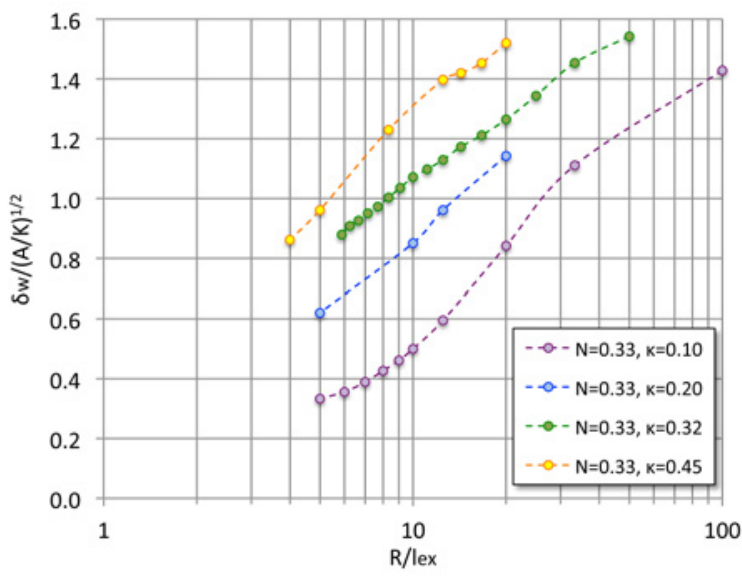

Fig. 8. Domain wall width for a spherical particle vs. $R / \ell_{e x}$ for different hardness parameter

\section{References}

1. O. Bottausio, M. Chiampi, A. Manzin, IEEE Trans. Magn. 44, 3149 (2008)

2. T.L. Gilbert, IEEE Trans. Magn. 40, 3443 (2004)

3. J.M.D. Coey, Magnetism and Magnetic Materials, Cambridge University Press (2011)

4. W. F. Brown, Jr., Micromagnetics, Wiley, New York (1963)

5. D Suess, V Tsiantos, T Schrefl, J Fidler, W Scholz, H Forster, R Dittrich, J.J Miles, J Magn. Magn. Mat. 248, 298 (2002)

6. H. Kronmüller, M. Fänhle, Micromagnetism and microstructure of ferromagnetic solids, Cambridge University Press (2003) 